\title{
MetaBoot: A machine learning framework of taxonomical biomarker discovery for different microbial communities based on metagenomic data
}

Xiaojun Wang, Xiaoquan Su, Xinping Cui, Kang Ning

As more than $90 \%$ of species in a microbial community could not be isolated and cultivated, the metagenomic methods have become one of the most important methods to analyze microbial community as a whole. With the fast accumulation of metagenomic samples and the advance of next-generation sequencing techniques, it is now possible to qualitatively and quantitatively assess all taxa (features) in a microbial community. A set of taxa with presence/absence or their different abundances could potentially be used as taxonomical biomarkers for identification of the corresponding microbial community's phenotype. Though there exist some bioinformatics methods for metagenomic biomarker discovery, current methods are not robust, accurate and fast enough at selection of nonredundant biomarkers for prediction of microbial community's phenotype. In this study, we have proposed a novel method, MetaBoot, that combines the techniques of mRMR (minimal redundancy maximal relevance) and bootstrapping, for discover of nonredundant biomarkers for microbial communities through mining of metagenomic data. MetaBoot has been tested and compared with other methods on well-designed simulated datasets considering normal and gamma distribution as well as publicly available metagenomic datasets. Results have shown that MetaBoot was robust across datasets of varied complexity and taxonomical distribution patterns and could also select discriminative biomarkers with quite high accuracy and biological consistency. Thus, MetaBoot is suitable for robustly and accurately discover taxonomical biomarkers for different microbial communities. 
2 Xiaojun Wang ${ }^{1,2}$, Xiaoquan Su${ }^{1,3}$, Xinping Cui ${ }^{1,4, *}$, Kang Ning ${ }^{1,2,3, *}$

$3{ }^{1}$ Bioinformatics Group of Single Cell Center, Shandong Key Laboratory of Energy Genetics and CAS

4 Key Laboratory of Biofuels, Qingdao Institute of Bioenergy and Bioprocess Technology, Chinese

5 Academy of Sciences, Qingdao 266101, Shandong Province, People's Republic of China

6

$7 \quad{ }^{2}$ University of Chinese Academy of Sciences, Beijing 100864, People's Republic of China

$9 \quad{ }^{3}$ CUDA Research Center, Qingdao Institute of Bioenergy and Bioprocess Technology, Chinese

10 Academy of Sciences, Qingdao 266101, Shandong Province, People's Republic of China 11

${ }^{4}$ Department of statistics, University of California, Riverside. Riverside, California, USA 13

14 *Corresponding author:

15 E-mail: ningkang@qibebt.ac.cn

$16 \quad$ xinping.cui@ucr.edu

17

18 Email-address for all authors:

19 Xiaojun Wang: wang_xj@qibebt.ac.cn

20 Xiaoquan Su: suxq@qibebt.ac.cn

21 Xinping Cui: xinping.cui@ucr.edu

22 Kang Ning: ningkang@qibebt.ac.cn 


\section{Introduction}

24 The approximate estimation of microbial cells on earth is $10^{30}$ (Proctor, 1994), which is huge, and a 25 large number of novel genes with useful functions might be contained within the genomes of these unknown communities of microbes. However, it was estimated that more than $90 \%$ of species in the microbial communities are unknown and uncultivable (Jurkowski, Reid \& Labov, 2007). Therefore, the traditional processes for isolation and cultivation of microbes are not applicable for the analyses of many microbial communities. Based on the development of Next Generation Sequencing (NGS), metagenomic method become one of the important methods that could provide direct access to genomes of as yet uncultivated microorganisms in native environments (Eisen, 2007). And metagenomics makes it possible to better understand microbial diversity as well as their functions. Metagenomics has become an increasingly popular research area when considering its diverse and multiplicity of metagenomics and its potential applications in environmental sciences, bioenergy and human health.

One of the most broadly applicable and successful means of translating molecular and genomic data into applications such as clinical practice (Segata et al., 2011) and environmental monitoring (Lam \& Gray, 2003) is the identification of biomarker. Comparisons among different types of tissues or samples have highlighted the importance of detecting novel subtypes of a disease or determining the subtype of a new sample (Golub et al., 1999, Tothill et al., 2008). In any genomic dataset, identifying the most biologically informative features which can differentiate two or more sets of samples remains an obstacle, and for metagenomic biomarkers this is particularly true.

Other than the challenges associated with high-dimensional data which includes different meta data or data type, metagenomic analysis additionally presented their own specific issues, including sequencing errors, chimeric reads (Swan et al., 2002, Wooley \& Ye, 2010) and complex underlying biology 
(multiple species and their uniqueness, relative abundances, complex functions, etc.). Remarkable inter-subject variability would usually present a profound property of many microbial communities as well, which has made biomarker identification a big hurdle as well. For instance, both environmental and human microbiomes might be subjected to a long tail distribution of rare organisms (Liao et al., 2011, Pedrós-Alió, 2006). Therefore, robust and efficient bioinformatics tools that could ensure the reproducibility of biomarker identification from metagenomic data, which is crucial for its applications, are needed. What is more, as mention in Segata, N. et al.(Segata et al., 2011), elucidating the biological consistency and roles of selected biomarker, especially non-redundant biomarkers, is a crucial step to understand the underlying mechanisms of community-community or host-community interactions.

A number of methods have been developed for comparison of different metagenomic samples from different angles. Firstly, there are methods that could assess whether, other than how communities differ. DOTUR (Schloss \& Handelsman, 2005) and SONS (Schloss \& Handelsman, 2006) cluster sequences into operational taxonomic units (OTUs), and by estimating the diversity of a microbial population, provide a coarse measure for comparing different communities. TreeClimber (Schloss \& Handelsman, 2006), UniFrac (Lozupone \& Knight, 2005) and Meta-Storms (Su, Xu \& Ning, 2012) compare sets of metagenomics in a phylogenetic context. Secondly, there are tools for comparing two sets of samples. MEGAN (Huson et al., 2007) is a metagenomic analysis tool providing a graphical interface that allows users to compare the taxonomic composition of samples, with additions for phylogenetic comparisons and statistical analyses. MEGAN, however, can only compare single pairs of metagenomic samples, which is also the case with STAMP (Parks \& Beiko, 2010). Thirdly, statistical model based methods were developed for the comparison of samples. MG-RAST (Meyer et al., 2008), ShotgunFunctionalizeR (Kristiansson, Hugenholtz \& Dalevi, 2009), Mothur (Schloss et al., 2009) and METAREP (Goll et al., 2010) all compare metagenomic samples through standard statistical tests. However, none of these methods directly identify biological features responsible for group relationships (Gower, 1966). 
The identification of biomarkers for metagenomic data could illustrate the reason for metagenomic sample differences. There are two general approaches for metagenomic biomarker discovery: bottomup and top-down. The bottom-up method is the one that tested each taxa and selected ones that would led to the variations between groups. Typical bottom-up methods include Wilcoxon rank-sum test (Wilcoxon) (Bauer, 1972). The top-down method is based on statistical analysis of the overall distribution of taxon in the metagenomic samples. Currently, Metastats (White, Nagarajan \& Pop, 2009) and LEfSe (Segata et al., 2011) are the only two available methods that explicitly apply statistical assessment of metagenomic difference for metagenomic biomarker discovery. LEfSe further considered biological relevance, biological consistency and effect size estimation of predicted biomarkers. As pointed out by LEfSe (Segata et al., 2011), to ensure reproducibility of biomarker identification from metagenomic data, robust statistical tools are needed, which is also critical for clinical applications. However, none of the aforementioned two methods have addressed the issue of robustness. In addition, redundancy is a serious issue for metagenomic data analysis, especially for biomarker discovery. Taxonomically, as microbial community is dynamic, it is very common that there exist many similar strains as well as multiple similar mutants of the same strain. However, to maximize the power of biomarkers for clinical diagnostic application, it is desirable to find biomarkers that are both distinguishable and representative. Therefore, biomarkers from the same strain and its mutants or from similar strains are considered as redundant biomarkers since they contain similar genetic and/or clinical information. Note that redundancy in biomarker discovery from gene expression data is less of an issue in that even though two or more genes might be similar, they might play significantly different roles in the biological system (biological importance). Additionally, the evolutionary relationship among similar genes might not be that close enough to treat them as the redundant biomarker.

In this work, we present a top-down strategy, MetaBoot, which uses mRMR (Ding \& Peng, 2005) and Bootstrap for feature selection from microbial community samples. Strategically, it is a top-down approach in the sense that it first analyzed the overall structure of the microbial community, and then summarized such property for biomarker identification. The MetaBoot framework is based on 
102 taxonomical profiles generated from the microbial community's 16S rRNA gene sequences. It selects

103 discriminative features as candidate features through bootstrap resampling. This general procedure is

104 simple in principle, yet it is significantly different from previous biomarker discovery methods: It is

105 different from previous metagenomic biomarker methods in the final results which would be a set of

106 non-redundant and informative features (genes) selected by mRMR, rather than a complex taxonomy

107 structure or a set of many biologically redundant features. And it introduces bootstrap resampling

108 procedure to ensure the robustness and reproducibility.

109

110 MetaBoot has been put to test and compared with other methods on well-designed simulated

111 metagenomic datasets with known biomarkers and realistic taxonomical distribution properties. Results

112 have shown that MetaBoot was robust for biomarker discovery across datasets of varied complexity

113 and taxonomical distribution patterns. On real oral and soil metagenomic datasets, MetaBoot could also

114 select discriminative biomarkers with high specificity and clear biological meaning.

\section{Materials and Methods}

116 Data Description

\section{Synthetic datasets}

118 We generated three collections of artificial datasets in order to compare MetaBoot with other methods.

Synthetic dataset $\boldsymbol{S} \boldsymbol{1}$ (normal dataset). To demonstrate the ability of our method to select features with lower redundancy compared with LEfSe, Metastats and Wilcoxon, we built synthetic dataset $S 1$ (Figure 1). Dataset $S 1$ include 2 classes with three subclasses each, and each subclass has 20 samples.

123 For each sample, there are 10 feature groups (with 10 features in each group) for positive biomarkers and 1 feature group (with 900 features) for negative biomarkers. Therefore there are 1,000 features and 
normal distribution as described in Figure 1. Dataset $S 1$ has two properties: firstly, for positive marker groups, features in class 1 and class 2 have clear difference in mean values, and the between-class differences are larger than between-subclass differences. Secondly, there are feature-to-feature variations within the same feature group due to random distribution function. Nevertheless, features within the same feature groups are considered as redundant features in the dataset.

In the process of analyzing real data (16S rRNA sequencing data from oral samples), we found that the distribution of many features (taxas) conformed mixture of normal and gamma distribution or gamma distribution (Figure 3). For some real data, the defects of measurement could lead to this result. But there possibly exist features whose distribution conform gamma distribution in real data. Therefore, we built the synthetic dataset $S 2$ (mixture dataset) and $S 3$ (gamma dataset). There are two important parameters, shape and rate, in gamma distribution and both parameters are positive real numbers.

Because the change of shape parameter has a greater impact upon the shape of gamma distribution than that of rate parameter, most of the positive markers among subclasses have different shape parameter. The biomarkers that could differentiate "class 1" and "class 2" samples were the subject of biomarker identification.

Synthetic dataset $\boldsymbol{S} 2$ (mixture dataset). The detailed parameter settings were shown in Table 1. For positive marker groups 1-5, features in class 1 and class 2 have clear difference in shape values. And for positive marker groups 6-10, features in class 1 (gamma distribution) and class 2 (normal distribution) have clear difference in mean values (The mean and $s d$ values of features in class 2 are determined based on mean and $s d$ values from corresponding features in class 2 with gamma distribution). Dataset $S 2$ (mixture dataset) has three properties: firstly, for positive marker groups, features in class 1 and class 2 have clear difference in shape or mean values, and the between-class differences are larger than between-subclass differences. Secondly, for negative marker groups, there is no difference between classes in mean values. Thirdly, there are feature-to-feature variations within the same feature group due to random distribution function. Nevertheless, features within the same feature 
153 groups are considered as redundant features in the dataset $S 2$. The biomarkers that could differentiate 154 "class 1" and "class 2" samples were the subject of biomarker identification.

155

156

157

158

159

160

161

162

163

164

165

166

167

168

169

170

171

172

173

174

175

176

177

178

Synthetic dataset $\boldsymbol{S 3}$ (gamma dataset). The detailed parameter settings were shown in Table 2.

Dataset $S 3$ (gamma dataset) has three properties: firstly, for positive marker groups, features in class 1 and class 2 have clear difference in shape values, and the between-class differences are larger than between-subclass differences. Secondly, for negative marker groups, there is no difference between classes in shape values. Thirdly, there are feature-to-feature variations within in the same feature group due to random function. Nevertheless, features within the same feature groups are considered as redundant features in the dataset $S 3$. The biomarkers that could differentiate "class 1" and "class 2 " samples were the subject of biomarker identification.

\section{Real datasets}

Oral dataset1: oral samples from Huang S, et al. (Huang et al., 2014). Supragingival plaques were sampled from fifty volunteers recruited at Day-21, Day 0 (Baseline) and Day-21 (different from the previous Day-21). In this experiment, based on these three time-points, we have generated two groups of samples: (1) The "oral hygiene phase” (Day-21 to Day 0) group, also referred to as EG group. (2) The "experimental gingivitis phase" (Day 0 to Day 21) group, also referred to as NG group. Totally, oral dataset1 includes 100 samples (50 samples for each group). For each of samples, 16S rRNA gene sequencing data were generated, and microbial community structure were then analyzed by Mothur (Schloss et al., 2009) for taxa and their relative abundances in the sample. The biomarkers that could differentiate "oral hygiene phase" and "experimental gingivitis phase" were the subject of biomarker identification.

Oral dataset2. Oral dataset2 from Human Microbiome Project (HMP, http://www.hmpdacc.org) includes 812 samples in which 344 samples are from saliva and other 468 samples are from subgingival plaque. And Oral dataset2 includes 44, 69 and 96 features at order, family and genus level, 
179 respectively. For each of samples, 16S rRNA sequencing data were generated, and microbial

180 community structure were then analyzed by Parallel-Meta (Su, Xu \& Ning, 2014) for taxa and their

181 relative abundances in the sample. The biomarkers that could differentiate "saliva" and "subgingival

182 plaque" origins were the subject of biomarker identification.

183

184

185

186

187

188

189

190

191

192

193

Soil dataset: soil samples from Caporaso JG, et al. (Caporaso et al., 2011). Soil dataset includes 14 samples for which 7 samples were collected from two kinds of soil environment each with different $\mathrm{pH}$ values $(\mathrm{pH}=4.9$ and 8.4 , respectively). For each of samples, $16 \mathrm{~S}$ rRNA sequencing data were generated, and microbial community structure were then analyzed by Parallel-Meta for taxa and their relative abundances in the sample. The biomarkers that could differentiate " $\mathrm{pH}=4.9$ " and " $\mathrm{pH}=8.4$ " were the subject of biomarker identification.

\section{MetaBoot algorithm}

The overall MetaBoot algorithm includes (1) normalization step, (2) first feature selection step, (3) bootstrap and feature selection step and (4) feature rank step. Figure 2 is the flow chart of MetaBoot process.

\section{Data normalization}

To account for difference of read counts across multiple samples in magnitude, we pre-process the data and convert the raw read counts into relative abundances with per-sample normalization to sum to one (raw read counts/total counts in each sample). And the feature whose $80 \%$ values are 0 should be deleted. Notice that for each of samples from real datasets, 16S rRNA sequencing data analyzed by Parallel-Meta (Su, Xu \& Ning, 2014) for taxa and their relative abundances in the sample. Every taxa's relative abundances were already normalized by Parallel-Meta as default setting.

Dataset is discretized before input into mRMR feature selection process. The discretization of the data into categorical data not only helps reduce the substantial noise contained in raw data but also increases the power of mRMR method selecting discriminative features. In our method, we use the method 
mentioned in previous work (Ding \& Peng, 2003) to discretize our data into categorical data. Each feature (also called attribute or variable) of data is discretized using its $\mu$ (mean) and $\sigma$ (standard deviation): any data larger than $\mu+\sigma / 2$ are converted into 1 ; any data smaller than $\mu-\sigma / 2$ are converted into -1 ; otherwise, data are converted into 0 .

\section{Main process}

The input dataset for feature selection are required to be normalized data.

(1) In the first feature selection step, a number of candidate features (Parameter 1, M. $M$ represents the number of features in the first feature selection step.) would be selected by mRMR that could discriminate different samples, but might include many redundant features. Therefore, we employed the following two steps to minimize redundancy. The dataset which included $M$ selected features would be used in the subsequent steps.

(2) The bootstrap process (parameter 2, B. B represents the number of bootstrapping process in this step) is employed to eliminate negative markers and redundant positive markers. Here we have implemented bootstrapping with a principle that the number of samples in each subclass (For example, subclass 1 in Figure 1) (or class when the original data has no subclasses) of the bootstrapped dataset must be equal to that in the same subclass (or class) of original dataset. In other words, we require that the new dataset generated by bootstrapping has the same structure as original dataset. The only difference between original datasets and bootstrapped datasets would be that some samples may appear more than once and some samples may not appear in new dataset.

(3) At the feature rank step, the top $\mathrm{N}$ (Parameter 3, $M^{\prime} . M^{\prime}$ represents the number of final features selected) discriminative features from each bootstrapped dataset will be selected by mRMR. All selected features were ranked according to the number of occurrences. $M$ ' of the top ranked features will be selected as our final biomarkers. 
230 The 3 parameters involved in this process could be adjusted according to each project's requirement

231 and specific metagenomic data. Yet it should be emphasized that $M$ must be greater than $M^{\prime}$.

\section{Assessment methods for comparison of different biomarker identification methods}

To evaluate and compare different biomarker identification methods, we have defined the redundancy rate, non-redundancy rate, error rate, and classification accuracy as follows:

$$
\begin{aligned}
& \text { Redundancy rate }=\frac{\# \text { redundancy features }}{\# \text { features selected }} * 100 \% \\
& \text { Non - redundancy rate }=1-\text { Redundancy rate } \\
& \text { Error rate }=\frac{\# \text { negative features }}{\# \text { features selected }} * 100 \% \\
& \text { Classification accuracy }=\frac{\# \text { samples correctly classified }}{\# \text { samples in testing dataset }} * 100 \%
\end{aligned}
$$

\section{Implementation and availability of the method}

237 The MetaBoot method is implemented in MATLAB. The software and simulated data that used in this

238 paper could be found online at http://www.computationalbioenergy.org./metaboot.html. The original

239 mRMR codes are wrapped for feature selection module within MetaBoot. Therefore MetaBoot cannot

240 be used for commercial application without consent from the author of mRMR and MetaBoot.

\section{The selection standard or parameter setting for different methods}

242 LEfSe: Selecting the features with (1) lower p-value and (2) higher effect size (Segata et al., 2011).

243 About parameter setting, we used the default parameters.

244 Metastats: Selecting the features with lower p-value (White, Nagarajan \& Pop, 2009). About

245 parameter setting, we used the default parameters. 
246 Wilcoxon: Selecting the features with lower p-value.

247 MetaBoot: Selecting the features with higher bootstrapping frequency.

248 LIBSVM: optimizing the parameters by using the script (easy.py) to achieve the best classification 249 accuracy. Therefore, for different datasets, the parameters might be different.

250 mRMR: the feature selection scheme we used was MID (Mutual Information Difference) (Ding \& 251 Peng, 2005).

\section{Results and Discussions}

253 One bottleneck for assessment of the effectiveness of MetaBoot for identifying biomarkers from 254 microbial community data is the lack of "ground truth". To overcome this problem, we have first 255 analyzed taxonomical distribution properties of real metagenomic samples, and generated sets of 256 synthetic datasets with known ground truth biomarkers and distribution properties learned from real 257 data. Secondly, we have analyzed the effects of different parameters on MetaBoot results, using 258 synthetic datasets. Thirdly, we have evaluated the overall performance of MetaBoot by comparing with 259 other methods. Finally, we have assessed the effectiveness of MetaBoot on real datasets.

\section{Taxonomical distribution patterns of real metagenomic samples}

In this work, we used oral dataset1 to analyze distribution properties of real metagenomic samples. the taxonomical distribution of microbial community conform to normal distribution (Segata et al., 2011). Therefore, we have generated synthetic datasets $S 1$ (Normal dataset) based on normal 
270 distributions (see Materials and Methods for details).

272 Secondly, we have evaluated the taxonomical distribution properties for taxa at genus level as features.

273 Based on the analysis of the distribution of oral microbial community dataset (dataset described in

274 Materials and Methods), we observed that the distribution of a couple of features (about 10\% taxa)

275 conformed a mixture of normal and gamma distribution. For example, taxon Leptotrichia and its

276 mixture of distributions were shown in Figure 3a-c. Therefore, we generated synthetic dataset $S 2$

277 (Mixture dataset) based on the mixture of normal and gamma distribution (see Materials and

278 Methods for details).

279

280 Thirdly, we have found that the distribution of over $40 \%$ taxa (one example for taxon Actinonyces

281 shown in Figure 3d) in oral dataset1 conformed gamma distribution tested by the Kolmogorov-

282 Smirnov Tests (Birnbaum \& Tingey, 1951) (function ks.test in R). The p-values of KS test were 0.78

283 and 0.93, respectively, for the two sets (EG and NG) of samples. Therefore, we generated synthetic

284 dataset $S 3$ (Gamma dataset) based on gamma distribution (see Materials and Methods for details).

\section{MetaBoot analysis}

286 Here we chose taxa at genus level for analysis, which could be accurately identified by Mothur 287 (Schloss et al., 2009) and Parallel-Meta (Su, Xu \& Ning, 2014) software based on the OralCore 288 (Griffen et al., 2011) and GreenGenes (DeSantis et al., 2006) databases, and are detailed enough and 289 widely used for differentiating ingredients of communities. For each synthetic datasets (S1, S2 and S3), 290 we aimed to differentiate "class 1" and "class 2" samples using MetaBoot (see Materials and 291 Methods for details).

293 The MetaBoot process includes 3 major steps: first feature selection step, bootstrap and feature 294 selection step, feature rank step. Throughout the entire workflow of MetaBoot, 3 parameters $\left(M, M^{\prime}\right.$ 295 and $B$, see Materials and Methods for details) are most important for the quality of selected 
biomarkers.

For synthetic dataset $S 1, M$ was set to be 50, because we observed that when $M$ was set to 50, enough or all unique positive features could be obtained from 1000 features using mRMR (Figure 4a). Notice that we treated features from the same group as redundant features. After eliminating redundant features, the remaining features were unique features. If the unique features were from positive marker groups, we called those as unique positive features. Since synthetic dataset $S 1$ only includes 10 positive marker groups, we set $M^{\prime}$ to be 10 . In order to determine parameter $B$, we set a series gradient of the bootstrap process. We observed that when $B$ was more than 40 , the number of total unique features selected did not increase. Therefore, the $B$ value was set to 40 (Figure 4b). For synthetic dataset $S 2$ and $S 3$, we have observed similar patterns (see Supplementary Information for details). Therefore, in this work, parameters $M, B$ and $M$ ' were set to be 50, 40 and 10 respectively for all datasets.

\section{A comparison with current tools using synthetic data}

\section{Redundancy analysis based on synthetic datasets}

For comparison of 4 methods as regard to redundancy rate (Formula 1), non-redundancy rate (Formula 2) and error rate (Formula 3), we applied LEfSe, Metastats, a bottom-up method Wilcoxon rank-sum test (Wilcoxon) and our method (MetaBoot) on synthetic dataset $S 1$ (There are 10 positive biomarker groups and each group has 10 redundant biomarkers.), respectively. As shown in Figure 5, MetaBoot can select more non-redundant positive features than LEfSe, Metastats and Wilcoxon. Additionally, because the 100 positive markers have the same p-value (see Materials and Methods for details), Metastats in Figure 5 does not include error bars which indicate that the 10 selected features are from the same positive marker group (the first positive maker group). Therefore, Metastats could not eliminate redundant features when analyzing synthetic dataset $S 1$. 
321 For synthetic dataset $S 2$ (Table 3), MetaBoot could select at least 4 out of 10 non-redundant positive

322 biomarkers which was better than other three methods. For synthetic dataset $S 3$ (Table 3), LEfSe and

323 Metastats could only select less than 5 out of 10 non-redundant positive features on average. Both

324 Wilcoxon and MetaBoot outperformed LEfSe and Metastats in that they both can select at least 5 out

325 of 10 non-redundant positive biomarkers. Among these two, MetaBoot was slightly better than

326 Wilcoxon in selecting non-redundant positive markers.

327

328

When we further analyzed the differences between MetaBoot and mRMR, we could observe that MetaBoot had similar ability with mRMR in selecting unique positive markers based on synthetic dataset $S 1$ (see Figure S2 for details), $S 2$ (non-redundancy rate: $48.0 \% \pm 11.0$ ) and $S 3$ (non-redundancy rate: $53.6 \% \pm 10.1$ ). However, for most synthetic datasets from $S 1, S 2$ and $S 3, \mathrm{mRMR}$ usually had about 10\% error rate (Formula 3), while MetaBoot had much lower error rate (details of results not shown here).

\section{Robustness analysis based on synthetic datasets}

We have applied LEfSe, Metastats, Wilcoxon and MetaBoot on synthetic dataset $S 1, S 2$ and $S 3$ to study their robustness defined by their ability to differentiate positive and negative biomarkers, respectively. For each method, 100 features (equal to the number of redundant positive markers in synthetic datasets) were selected as biomarkers. Then the correctly detected biomarkers were counted. Results (Table 4 and Figure 6) have shown that MetaBoot and Wilcoxon method can detect larger number of correct biomarkers compared to other methods. Again though all four methods were shown to be robust on synthetic dataset $S 1$ (based on normal distribution), Wilcoxon and MetaBoot outperformed Metastats and LEfSe greatly on synthetic dataset $S 2$ (based on the mixture of normal and gamma distribution) and $S 3$ (based on gamma distribution), indicating the superiority of Wilcoxon and MetaBoot methods as regard to robustness.

As regard to robustness, MetaBoot was slightly better than mRMR in selecting positive markers based 
347 on synthetic dataset $S 1$ (see Figure S2 for details), $S 2$ (\#positive features: 67.4 \pm 3.6 ) and $S 3$ (\#positive

348 features: $80.2 \pm 3.0$ ). The built-in bootstrap process in MetaBoot might attribute to MetaBoot's

349 advantage in selecting more positive biomarkers compared to mRMR.

350 Classification accuracy analysis based on synthetic datasets

351 For comparison of different methods in classification accuracy (Formula 4), we have applied LEfSe,

352 Metastats, Wilcoxon and MetaBoot on synthetic dataset $S 3$ to select 10 features by each of the methods.

353 Then we used these 10 features to perform classification by utilizing Support Vector Machine (SVM)

354 implemented by LIBSVM (Chang \& Lin, 2011). The reason that we have not done classification based

355 on synthetic dataset $S 1$ was the large difference between 2 classes, making classification easy-proof by

356 all methods.

357

358 Each class has 60 samples in synthetic dataset $S 3$. We have performed 6-fold cross-validation to

359 estimate the classification accuracy. Therefore, in aforementioned formula, the average classification

360 accuracy is shown in Figure 7. The highest accuracy was obtained when using 10 features selected by

361 MetaBoot. We also observed that MetaBoot had the most stable classification performance (Figure

362 7).For synthetic dataset $S 2$, we obtained similar results (see Figure $\mathbf{S 3}$ for details).

\section{Biomarker identification based on real metagenomic datasets}

\section{Results on oral dataset1}

365 For this dataset, we aim to identify biomarkers that could differentiate "oral hygiene phase" and 366 "experimental gingivitis phase" from 16S rRNA gene sequencing data (details in Materials and 367 Methods). We have applied the same four methods on oral dataset1 to select 10 features. Biomarker 368 identification results were shown in Figure 8.

370 From Figure 8a, we observed that MetaBoot selected similar features ( 9 overlaps) with Wilcoxon, 
371 while only 4 and 5 feature overlapping with MetaBoot were found for LEfSe and Metastats,

372 respectively. As shown in Figure 8b, the 10 features selected by each of these methods could be

373 assigned to 6 to 7 phyla which are mostly overlapping. As shown in Figure 8, we observed that

374 Streptococcus were selected by MetaBoot, as well as LEfSe and Metastats. And Streptococcus was

375 linked with all kinds of oral problems (Munro \& Grap, 2004, Fitzgerald, 1960, Jenkinson \& Lamont,

376 2005). Therefore, Streptococcus can serve as biomarker to distinguish different samples and be used

377 for oral diagnosis (Bisno et al., 1997). Rothia were selected by Wilcoxon, as well as LEfSe. Rothia is

378 part of the normal community of microbes residing in the mouth. And previous work found Rothia in 3\%

379 of isolates of nitrate-reducing bacteria from the mouth (Doel et al., 2005).

381 To compare the discriminations accuracy of 10 features selected by different methods, we performed

382 classification by LIBSVM (Chang \& Lin, 2011). Each class in oral dataset1 has 50 samples. And we

383 did 5-fold cross-validation (40 samples are used as training datasets) to estimate the classification

384 accuracy. The classification results were shown in Figure 9, from which we could observe that

385 MetaBoot still had the highest accuracy and the most stable classification performance.

In order to evaluate the added value of bootstrap on mRMR (as realized in MetaBoot), we have also compared the results of mRMR vs. MetaBoot. Streptococcus mentioned above was linked with various oral problems. On the other hand, Cardiobacterium, selected only by mRMR as biomarker, was reported to be a rare cause of endocarditis (Han \& Falsen, 2005, Slotnick \& Dougherty, 1964), but it was not reported as oral related microbial biomarker in any known studies. The difference between mRMR and MetaBoot can be attributed to the bootstrap process included in MetaBoot. Therefore, apart from advantage in robustness, biomarkers selected by MetaBoot were considered more

394 biologically meaningful comparing to mRMR. (see Figure $\mathbf{S} 4$ for details)

\section{Results on oral dataset2}

396 For this dataset, we aim to identify biomarkers that could differentiate "saliva" and "subgingival 
397 plaque" from 16S rRNA sequencing data (details in Materials and Methods). We have applied the 398 same four methods on oral dataset2 at order, family and genus level to select 10 features, respectively. 399 As shown in vennplot (Figure 10a), at the level of genus and family, LEfSe, Wilcoxon and MetaBoot 400 had good coherence. Yet at order level, there were larger differences among results from different 401 methods. In addition, considering the complexity of the data, studies of microbial community 402 biomarker at order level would not be as reliable as on genus and family levels and seldom used.

403 Therefore, we only focused on the difference among different methods at the level of genus and family. 404 At the genus level (Figure 10a genus), Peptostreptococcus (Figure 10b), which was selected by 405 MetaBoot, has been isolated from a wide range of human oral infections (Downes \& Wade, 2006) and 406 implicated in human gingivitis and periodontitis (Riggio \& Lennon, 2003). At family level (Figure 10a 407 family), Spirochaetaceae (Figure 10b) was selected by MetaBoot but not other method. It was also interesting to observe that all oral spirochetes (belonging to Spirochaetaceae family) were classified in the genus Treponema (Chan and McLaughlin, 2000), and Treponema was reported to be associated with periodontal diseases (Chan \& McLaughlin, 2000, Sela, 2001). But for Propionibacteriaceae (Figure 10b), which was selected by LEfSe and Wilcoxon, though this species could be isolated from normal, gingivitis and periodontitis sample with small amount (Riggio et al., 2011), there was few report about the relationship between oral disease and Propionibacteriaceae. Therefore, these results on real oral samples have clearly shown the advantage of MetaBoot on discovery of biologically meaningful biomarkers.

\section{Results on soil samples}

417 For this dataset, we aim to identify biomarkers that could differentiate " $\mathrm{pH}=4.9$ " and " $\mathrm{pH}=8.4$ " from 418 16S rRNA sequencing data (details in Materials and Methods). Unlike two oral datasets, each class in 419 soil dataset only has 7 samples. Therefore, we focused on the different features selected by different 420 methods not the distribution properties of features (The sample size is small for distribution analysis.).

421 Due to the complexity of soil microbial community samples, we chose taxa at phylum level for 422 analysis. 
424 When we performed classification by LIBSVM (Chang and Lin, 2011), the classification accuracy was

425

426

427

428

429

430

431

432

433

434

435

436

437

438

439

440

\section{Conclusions}

442

443

444

445

446

447

448

always $100 \%$ regardless of either 5 or 10 features (selected by the four different methods) we used. For

soil dataset, features selected by the four different methods all had distinguishing ability to identify

different samples. However, biological explanation of features selected by the four different methods needed further research.

Based on the above results for soil samples, we could observe that features selected by the four different methods were quite different (Figure 11a), yet most of these features had distinguishing power to identify different samples. Further investigation and interpretation of these features might provide more biological insights for the underline functionality of microbial community.

As shown in Figure 11b, Burkholderiaceae (selected by the four methods) was enriched in acidic condition $(\mathrm{pH}=4.9)$. But when $\mathrm{pH}$ of soil was 8.5 , its relative abundance was low. And from different $\mathrm{pH}$ samples, the relative abundance of Burkholderiaceae had a significant difference ( $\mathrm{p}$-value = 0.00058). Therefore, Burkholderiaceae could serve as marker to differentiate soil samples with different $\mathrm{pH}$ values. Previous work has reported that Burkholderiaceae needs oxalic acid as its source of carbon (Garrity, Bell \& Lilburn, 2004), which partially support this finding.

The research in metagenomics becomes more and more popular as microbial communities were found to play important roles in many areas such as bioenergy, bioremediation and human health. The discovery of biomarker taxa for metagenomic datasets could facilitate identification of microbial community's phenotype, thus making them important for community identification and even monitoring of the host or environment within which the community live.

However, current metagenomic datasets lack "ground truth" of biomarkers, making it hard for the 
449

450

451

452

453

454

455

456

457

458

459

460

461

462

463

464

465

466

467

468

469

470

471

472

473

474

475

assessment of computationally predicted metagenomic biomarkers by various methods. A properly generated synthetic dataset with embedded "ground truth" and taxonomical distribution properties similar to those of real metagenomic samples could make such assessment fair and easy. In this study, we have evaluated taxonomical distribution properties for different microbial communities, and found that their taxonomical distributions follow either normal distribution, gamma distribution, or the mixture of normal and gamma distribution. Therefore in this work, synthetic datasets have been generated accordingly that could facilitate the assessment of biomarker identification methods.

We have proposed the MetaBoot method for metagenomic biomarker identification, which is a topdown method based on mRMR strategy and bootstrapping technique. The use of mRMR could reduce redundancies, while the use of bootstrapping could improve robustness of the MetaBoot method. It has been compared with two top-down methods (Metastats and LEfSe) and one bottom-up method (Wilcoxon rank-sum test) on simulated datasets, with results indicating that MetaBoot could identify more non-redundant biomarkers with high accuracy and robustness. On real oral and soil metagenomic datasets, it was also observed that MetaBoot could identify more reliable biomarkers for distinguish different types of microbial communities, showing that the results of MetaBoot were more biologically meaningful. Therefore, MetaBoot could serve well for metagenomic biomarker discovery.

Current taxonomical biomarker discovery methods still face several obstacles: Firstly most of them could identify biomarkers from only two groups of microbial communities, while biomarkers for a set of different groups could be more useful in several circumstances. Secondly the biomarker sets (with multiple biomarkers) might be useful for complex samples such as microbial community, yet none has been done on how such sets could be optimized. Thirdly with the advancement of whole genome sequencing, important functional biomarker identification using not only taxa but also genes would become feasible as well, yet current methods could not identify functional biomarkers well. All these analytical bottlenecks will be addressed in future development of MetaBoot and companion tools, and they in turn would help for better understanding of microbial communities and their impacts on our 
476 environment.

\section{Acknowledgements}

478 We thank Dr. Shi Huang for discussions about building MetaBoot, and Xingzhi Chang for comments 479 about writing codes.

480 References

481 Bauer, D.F., 1972. Constructing confidence sets using rank statistics. Journal of the American $482 \quad$ Statistical Association 67:687-690.

483 Birnbaum, Z., Tingey, F.H., 1951. One-sided confidence contours for probability distribution functions. $484 \quad$ The Annals of Mathematical Statistics 22:592-596.

485 Bisno, A.L., Gerber, M.A., Kaplan, E.L., Schwartz, R.H., 1997. Diagnosis and management of group A $486 \quad$ streptococcal pharyngitis: a practice guideline. Clinical Infectious Diseases 25:574-583.

487 Caporaso, J.G., Lauber, C.L., Walters, W.A., Berg-Lyons, D., Lozupone, C.A., Turnbaugh, P.J., Fierer, 488 N., Knight, R., 2011. Global patterns of 16S rRNA diversity at a depth of millions of sequences 489 per sample. Proceedings of the National Academy of Sciences 108:4516-4522.

490 Chan, E., McLaughlin, R., 2000. Taxonomy and virulence of oral spirochetes. Oral Microbiology and 491 Immunology 15:1-9.

492 Chang, C.C., Lin, C.J., 2011. LIBSVM: a library for support vector machines. ACM Transactions on $493 \quad$ Intelligent Systems and Technology 2:27.

494 DeSantis, T.Z., Hugenholtz, P., Larsen, N., Rojas, M., Brodie, E.L., Keller, K., Huber, T., Dalevi, D., 
Hu, P., Andersen, G.L., 2006. Greengenes, a chimera-checked 16S rRNA gene database and workbench compatible with ARB. Applied and Environmental Microbiology 72:5069-5072.

497

498

499

Ding, C., Peng, H., 2003. Minimum redundancy feature selection from microarray gene expression data, IEEE, pp 523-528.

Ding, C., Peng, H., 2005. Minimum redundancy feature selection from microarray gene expression data. Journal of Bioinformatics and Computational Biology 3:185-205.

Doel, J.J., Benjamin, N., Hector, M.P., Rogers, M., Allaker, R.P., 2005. Evaluation of bacterial nitrate reduction in the human oral cavity. European Journal of Oral Sciences 113:14-19.

Downes, J., Wade, W.G., 2006. Peptostreptococcus stomatis sp. nov., isolated from the human oral cavity. International Journal of Systematic and Evolutionary Microbiology 56:751-754.

Eisen, J.A., 2007. Environmental shotgun sequencing: its potential and challenges for studying the hidden world of microbes. Plos Biology 5:e82.

Fitzgerald, R.J., 1960. Demonstration of the etiologic role of streptococci in experimental caries in the hamster. Journal of the American Dental Association 61:9-13.

Garrity, G.M., Bell, J.A., Lilburn, T.G., 2004. Taxonomic outline of the prokaryotes. Bergey's Manual of Systematic Bacteriology. Springer, New York, Berlin, Heidelberg.

Goll, J., Rusch, D.B., Tanenbaum, D.M., Thiagarajan, M., Li, K., Methé, B.A., Yooseph, S., 2010. METAREP: JCVI metagenomics reports—an open source tool for high-performance comparative metagenomics. Bioinformatics 26:2631-2632.

Golub, T.R., Slonim, D.K., Tamayo, P., Huard, C., Gaasenbeek, M., Mesirov, J.P., Coller, H., Loh, M.L., Downing, J.R., Caligiuri, M.A., 1999. Molecular classification of cancer: class discovery 
and class prediction by gene expression monitoring. Science 286:531-537.

517 Gower, J.C., 1966. Some distance properties of latent root and vector methods used in multivariate analysis. Biometrika 53:325-338.

Griffen, A.L., Beall, C.J., Firestone, N.D., Gross, E.L., DiFranco, J.M., Hardman, J.H., Vriesendorp, B., Faust, R.A., Janies, D.A., Leys, E.J., 2011. CORE: a phylogenetically-curated 16S rDNA database of the core oral microbiome. Plos One 6:e19051.

Han, X.Y., Falsen, E., 2005. Characterization of oral strains of Cardiobacterium valvarum and emended description of the organism. Journal of Clinical Microbiology 43:2370-2374. Journal.

Huson, D.H., Auch, A.F., Qi, J., Schuster, S.C., 2007. MEGAN analysis of metagenomic data. Genome Research 17:377-386.

Jenkinson, H.F., Lamont, R.J., 2005. Oral microbial communities in sickness and in health. Trends in Microbiology 13:589-595. classroom (while it's still new). CBE-Life Sciences Education 6(4): 260-265.

534 Kristiansson, E., Hugenholtz, P., Dalevi, D., 2009. ShotgunFunctionalizeR: an R-package for 535 functional comparison of metagenomes. Bioinformatics 25:2737-2738.

536 Lam, P.K., Gray, J.S., 2003. The use of biomarkers in environmental monitoring programmes. Marine 
Pollution Bulletin 46:182-186.

538

539

540

541

542

543

544

545

546

547

548

549

550

551

552

553

554

555

556

557

Letunic, I., Bork, P., 2007. Interactive Tree Of Life (iTOL): an online tool for phylogenetic tree display and annotation. Bioinformatics 23:127-128.

Liao, L., Xu, X.W., Jiang, X.W., Wang, C.S., Zhang, D.S., Ni, J.Y., Wu, M., 2011. Microbial diversity in deep - sea sediment from the cobalt - rich crust deposit region in the Pacific Ocean. FEMS Microbiology Ecology 78:565-585.

Lozupone, C., Knight, R., 2005. UniFrac: a new phylogenetic method for comparing microbial communities. Applied and Environmental Microbiology 71:8228-8235.

Meyer, F., Paarmann, D., D'souza, M., Olson, R., Glass, E., Kubal, M., Paczian, T., Rodriguez, A., Stevens, R., Wilke, A., 2008. The metagenomics RAST server-a public resource for the automatic phylogenetic and functional analysis of metagenomes. BMC Bioinformatics 9:386.

Parks, D.H., Beiko, R.G., 2010. Identifying biologically relevant differences between metagenomic communities. Bioinformatics 26:715-721.

Pedrós-Alió, C., 2006. Marine microbial diversity: can it be determined? Trends in Microbiology 14:257-263.

Proctor, G.N., 1994. Mathematics of microbial plasmid instability and subsequent differential growth of plasmid-free and plasmid-containing cells, relevant to the analysis of experimental colony number data. Plasmid 32:101-130.

Riggio, M.P., Lennon, A., 2003. Identification of oral Peptostreptococcus isolates by PCR-restriction fragment length polymorphism analysis of 16S rRNA genes. Journal of Clinical Microbiology 41:4475-4479. 
558 Riggio, M.P., Lennon, A., Taylor, D.J., Bennett, D., 2011. Molecular identification of bacteria associated with canine periodontal disease. Veterinary Microbiology 150:394-400.

Schloss, P.D., Handelsman, J., 2005. Introducing DOTUR, a computer program for defining operational taxonomic units and estimating species richness. Applied and Environmental Microbiology 71:1501-1506.

Schloss, P.D., Handelsman, J., 2006. Introducing SONS, a tool for operational taxonomic unit-based comparisons of microbial community memberships and structures. Applied and Environmental Microbiology 72:6773-6779.

Schloss, P.D., Handelsman, J., 2006. Introducing TreeClimber, a test to compare microbial community

Segata, N., Izard, J., Waldron, L., Gevers, D., Miropolsky, L., Garrett, W.S., Huttenhower, C., 2011. structures. Applied and Environmental Microbiology 72:2379-2384.

Schloss, P.D., Westcott, S.L., Ryabin, T., Hall, J.R., Hartmann, M., Hollister, E.B., Lesniewski, R.A., Oakley, B.B., Parks, D.H., Robinson, C.J., 2009. Introducing mothur: open-source, platformindependent, community-supported software for describing and comparing microbial communities. Applied and Environmental Microbiology 75:7537-7541. Metagenomic biomarker discovery and explanation. Genome Biology 12:R60.

Sela, M.N., 2001. Role of Treponema denticola in periodontal diseases. Critical Reviews in Oral Biology \& Medicine 12:399-413.

578 Slotnick, I., Dougherty, M., 1964. Further characterization of an unclassified group of bacteria causing 
endocarditis in man: Cardiobacterium hominis gen. et sp. n. Antonie van Leeuwenhoek 30:261272.

Su, X., Xu, J., Ning, K., 2012. Meta-Storms: efficient search for similar microbial communities based on a novel indexing scheme and similarity score for metagenomic data. Bioinformatics 28:24932501. Visualization. Plos One 9.

Swan, K.A., Curtis, D.E., McKusick, K.B., Voinov, A.V., Mapa, F.A., Cancilla, M.R., 2002. Highthroughput gene mapping in Caenorhabditis elegans. Genome Research 12:1100-1105.

Tothill, R.W., Tinker, A.V., George, J., Brown, R., Fox, S.B., Lade, S., Johnson, D.S., Trivett, M.K., Etemadmoghadam, D., Locandro, B., 2008. Novel molecular subtypes of serous and endometrioid ovarian cancer linked to clinical outcome. Clinical Cancer Research 14:5198-5208.

594 Wooley, J.C., Ye, Y., 2010. Metagenomics: facts and artifacts, and computational challenges. Journal of Computer Science and Technology 25:71-81. 


\section{1}

The structure of synthetic dataset $S 1$ (dataset with normal distributions).

There is a 20 (samples)*10(features) matrix in each subclass and positive marker group. And data in each matrix was generated by the normal distribution function ( $r$ orm in R). More specifically, for group 1-5, the mean parameters for subclass 1, 2, 3 were randomly sampled from the vector (11, 12, 13 and 14); while the mean parameters for subclass 4, 5, 6 were randomly sampled from the vector $(17,18,19$ and 20). Data in group 6-10 were generated in a similar way by using these two vectors reversely. The 900 features in negative marker group all had the same mean value of 15 . All features had the same standard deviation ( $s d$ ) parameters. 


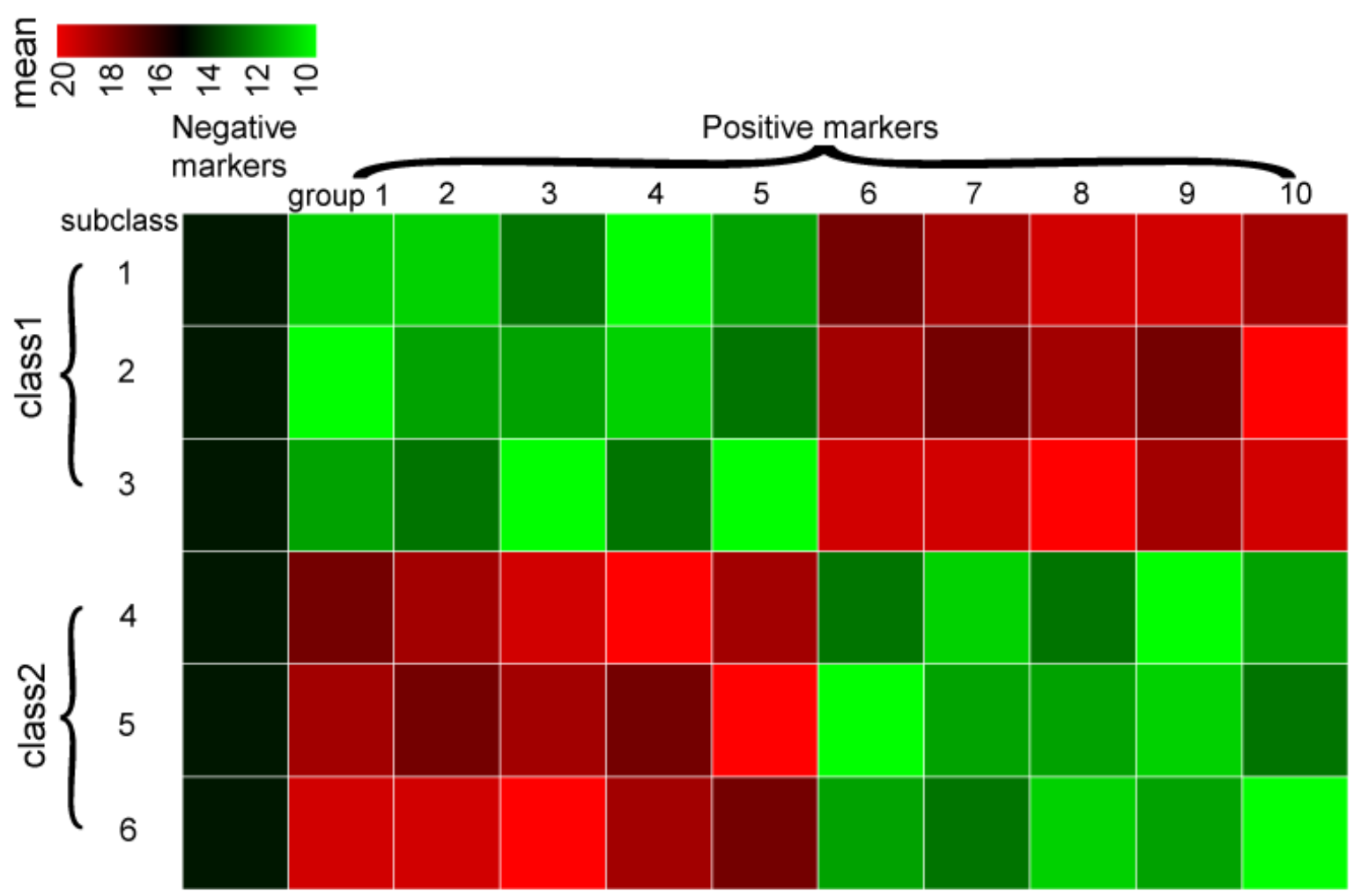


2

The flow chart of MetaBoot process.

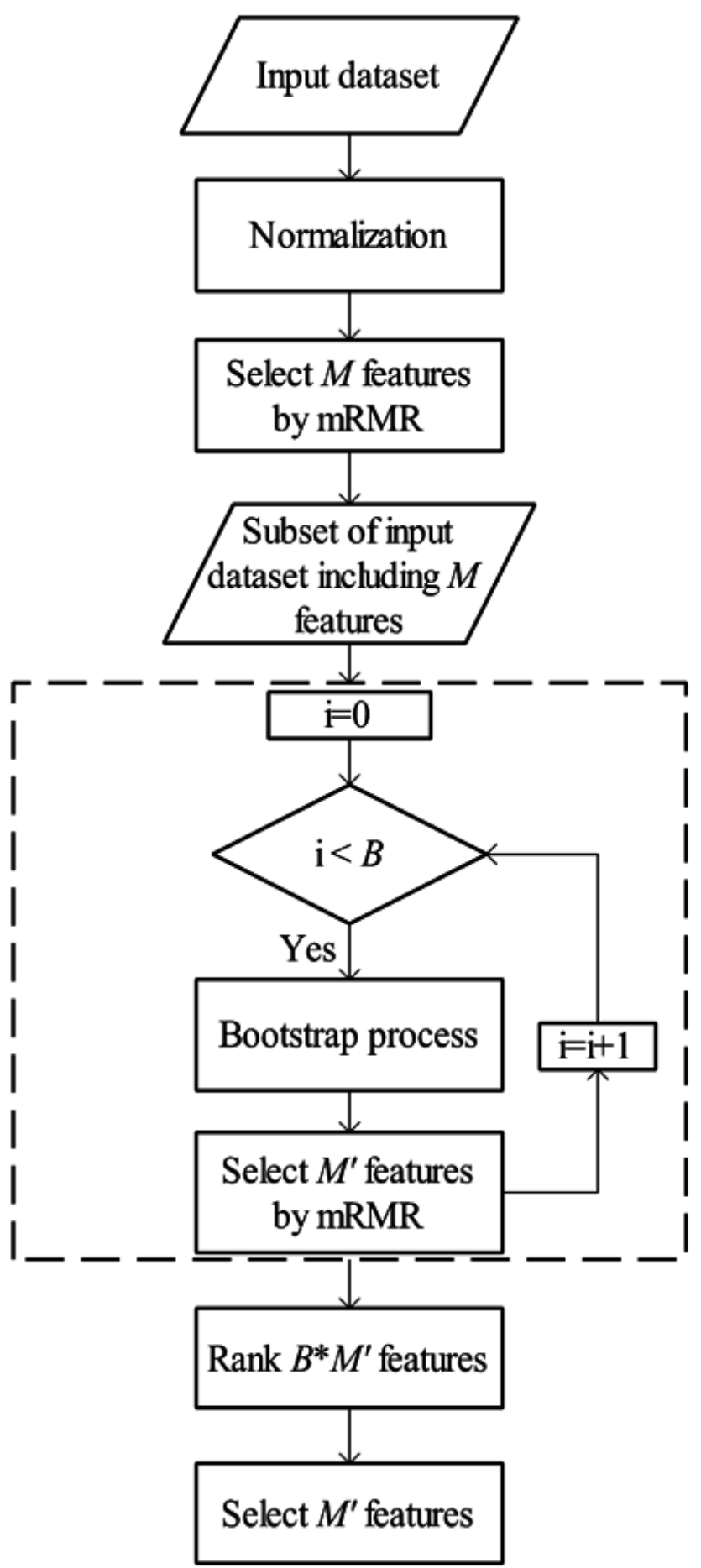




\section{Table $\mathbf{1}_{\text {(on next page) }}$}

The structure of synthetic dataset $S 2$ (dataset with mixture distributions).

In positive marker group, each square is a 25 (samples) $* 10$ (features) matrix in which each feature was generated by gamma (the red cells) or normal (the green cells) distribution function (generated by rgamma or rnorm in R). But in negative marker group, each square is a 25 (samples)*900(features) matrix in which each feature was also generated by normal distribution function. 


\begin{tabular}{|c|c|c|c|c|c|c|c|c|c|c|c|c|}
\hline \multirow{2}{*}{ class } & \multirow{2}{*}{$\begin{array}{l}\text { sub- } \\
\text { class }\end{array}$} & \multicolumn{10}{|c|}{ Positive marker group } & \multirow{2}{*}{$\begin{array}{c}\text { negative } \\
\text { marker group }\end{array}$} \\
\hline & & 1 & 2 & 3 & 4 & 5 & 6 & 7 & 8 & 9 & 10 & \\
\hline \multirow{8}{*}{ class 1} & \multirow{4}{*}{1} & shape & shape & shape & shape & shape & shape & shape & shape & shape & shape & mean \\
\hline & & 7.18 & 0.61 & 1.70 & 0.81 & 2.36 & 7.18 & 0.61 & 1.70 & 0.81 & 2.36 & 0.14 \\
\hline & & rate & rate & rate & rate & rate & rate & rate & rate & rate & rate & sd \\
\hline & & 44.38 & 71.12 & 517 & 79.70 & 316 & 44.38 & 71.12 & 517 & 79.70 & 316 & 0.06 \\
\hline & \multirow{4}{*}{2} & shape & shape & shape & shape & shape & shape & shape & shape & shape & shape & mean \\
\hline & & 6.98 & 0.51 & 1.80 & 0.91 & 2.46 & 6.98 & 0.51 & 1.80 & 0.91 & 2.46 & 0.14 \\
\hline & & rate & rate & rate & rate & rate & rate & rate & rate & rate & rate & $\mathrm{sd}$ \\
\hline & & 44.38 & 71.12 & 517 & 79.70 & 316 & 44.38 & 71.12 & 517 & 79.70 & 316 & 0.06 \\
\hline \multirow{8}{*}{ class2 } & \multirow{4}{*}{3} & shape & shape & shape & shape & shape & mean & mean & mean & mean & mean & mean \\
\hline & & 5.70 & 0.85 & 1.32 & 0.33 & 2.88 & 0.14 & 0.009 & 0.005 & 0.004 & 0.009 & 0.14 \\
\hline & & rate & rate & rate & rate & rate & $\mathrm{sd}$ & $\mathrm{sd}$ & $\mathrm{sd}$ & $\mathrm{sd}$ & $\mathrm{sd}$ & $\mathrm{sd}$ \\
\hline & & 44.38 & 27.40 & 210 & 91.20 & 507 & 0.06 & 0.007 & 0.002 & 0.006 & 0.06 & 0.06 \\
\hline & \multirow{4}{*}{4} & shape & shape & shape & shape & shape & mean & mean & mean & mean & mean & mean \\
\hline & & 5.60 & 0.75 & 1.22 & 0.43 & 2.98 & 0.13 & 0.010 & 0.004 & 0.003 & 0.010 & 0.14 \\
\hline & & rate & rate & rate & rate & rate & $\mathrm{sd}$ & $\mathrm{sd}$ & $\mathrm{sd}$ & $\mathrm{sd}$ & $\mathrm{sd}$ & $\mathrm{sd}$ \\
\hline & & 44.38 & 27.40 & 210 & 91.20 & 507 & 0.06 & 0.007 & 0.002 & 0.006 & 0.06 & 0.06 \\
\hline
\end{tabular}




\section{Table 2 (on next page)}

The structure of synthetic dataset S3 (dataset with gamma distributions).

In positive marker group, each square is a 20 (samples)*10(features) matrix in which each feature was generated by gamma distribution function (rgamma in R). But in negative marker group, each square is a 20 (samples)*300(features) matrix in which each feature was also generated by gamma distribution function. 


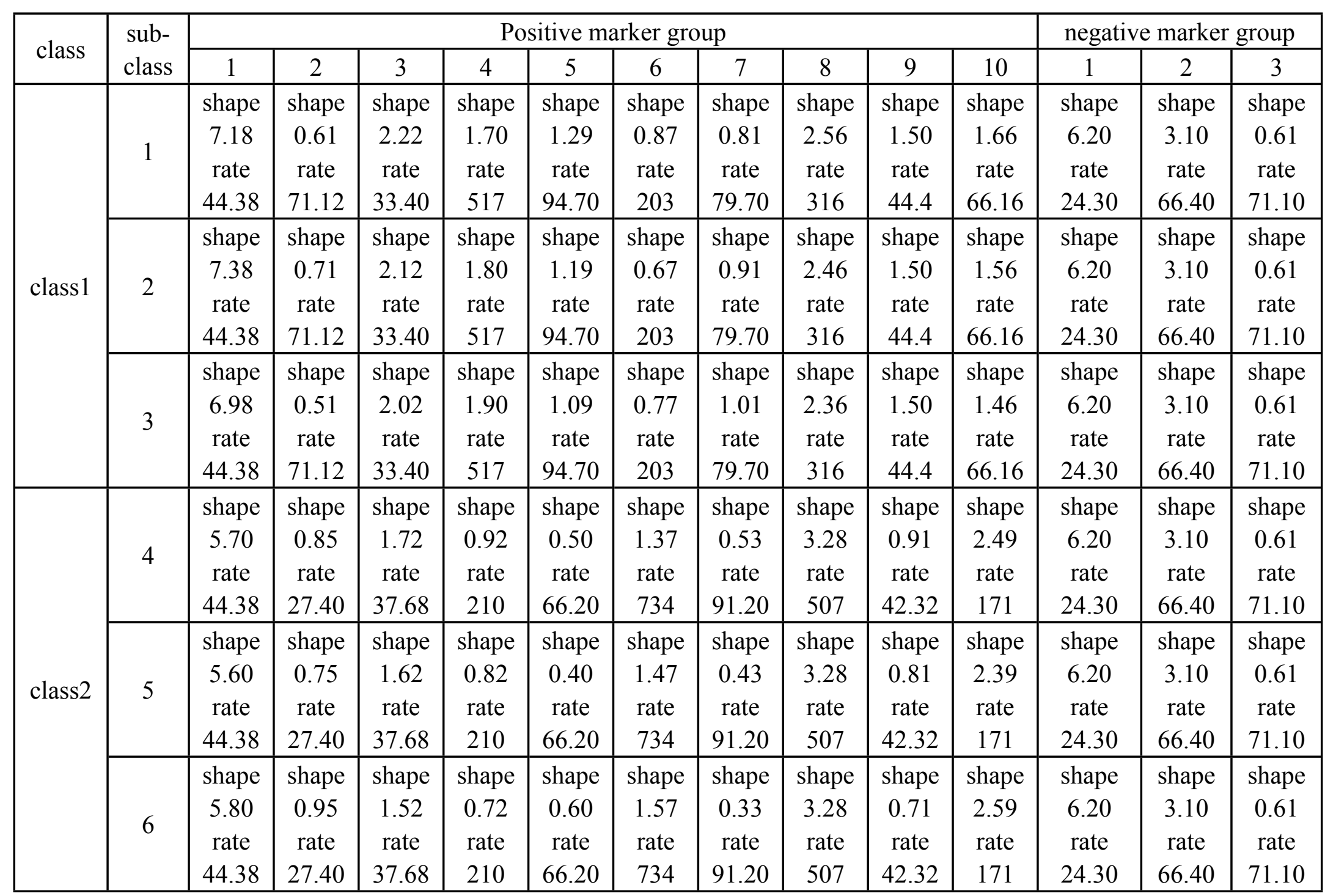




\section{3}

The distribution plot of taxon Leptotrichia and Actinonyces.

(a) The distribution of relative abundances for taxon Leptotrichia based on all samples in two categories (EG and NG) from Oral dataset1 (refer to Materials and Methods for details). The $x$-axis is relative abundance, and $y$-axis represents the number of samples. (b) The QQ plot of class EG (the red line in (a)) in taxon Leptotrichia. The p-value of Shapiro-Wilk Normality Test (Shapiro \& Wilk, 1965) is 0.93. (c) The QQ plot of class NG (the green line in (a)) in taxon Leptotrichia. The p-value of Shapiro-Wilk Normality Test is 0.02 . But the p-value of Kolmogorov-Smirnov Tests (Birnbaum \& Tingey, 1951) (KS test) is 0.46 when testing whether the distribution of class NG (the green line in (a)) in taxon Leptotrichia conform gamma distribution. (d) The distribution of EG and NG for taxa Actinonyces. The x-axis is relative abundance, and $y$-axis represents the number of samples. 

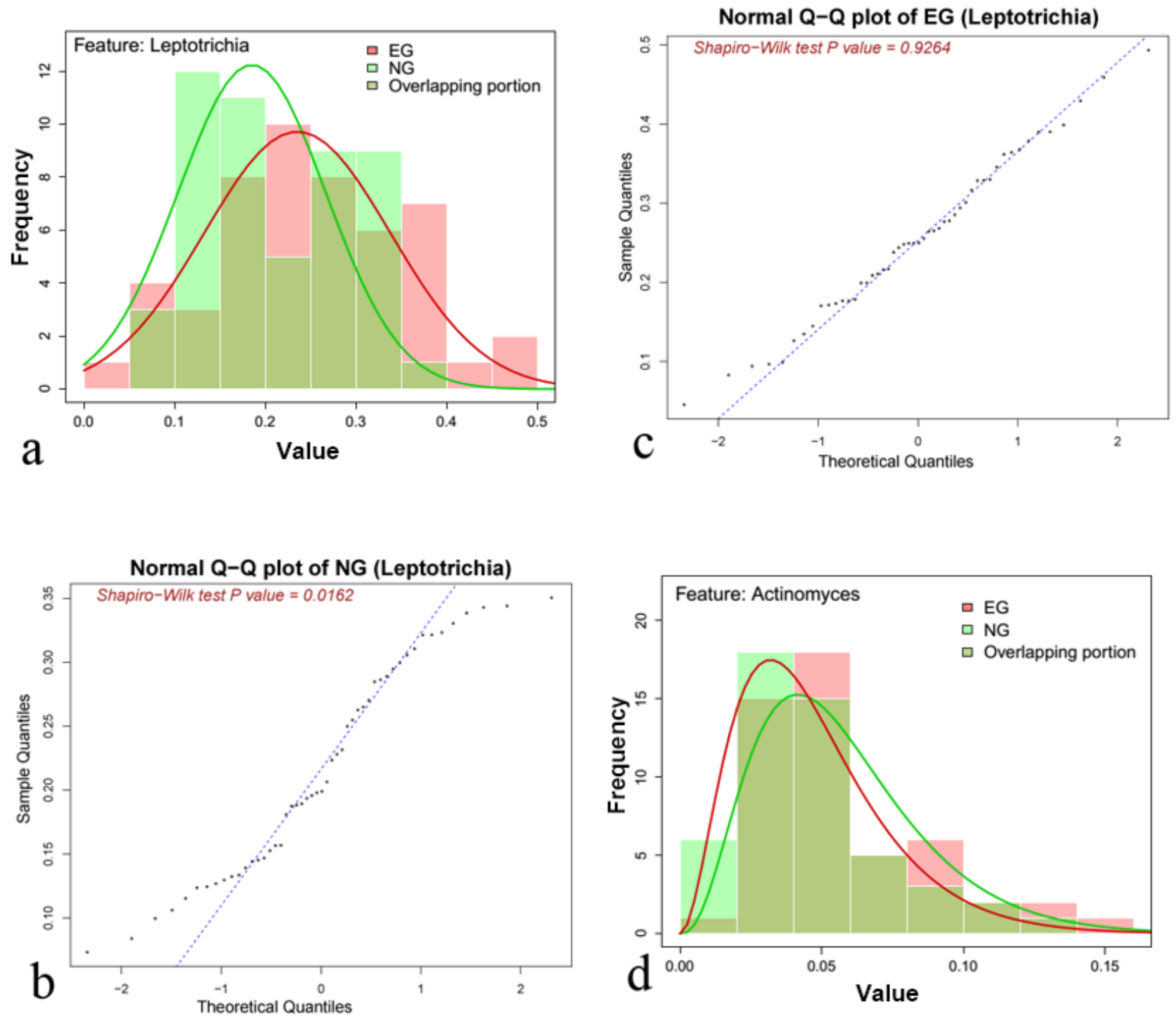
4

Plots for selecting $M$ and $B$ for MetaBoot analysis of synthetic data $S 1$.

(a) The $x$-axis is the values of $M$, and the $y$-axis is the number of unique positive features selected by mRMR for each given $M$. (b) The $x$-axis is the number of bootstraps $B$, and the $y$ axis is the number of unique features selected by all bootstrap processes. Both (a) and (b) considered different standard deviations ( $s d$ ) used in synthetic dataset $S 1$. 

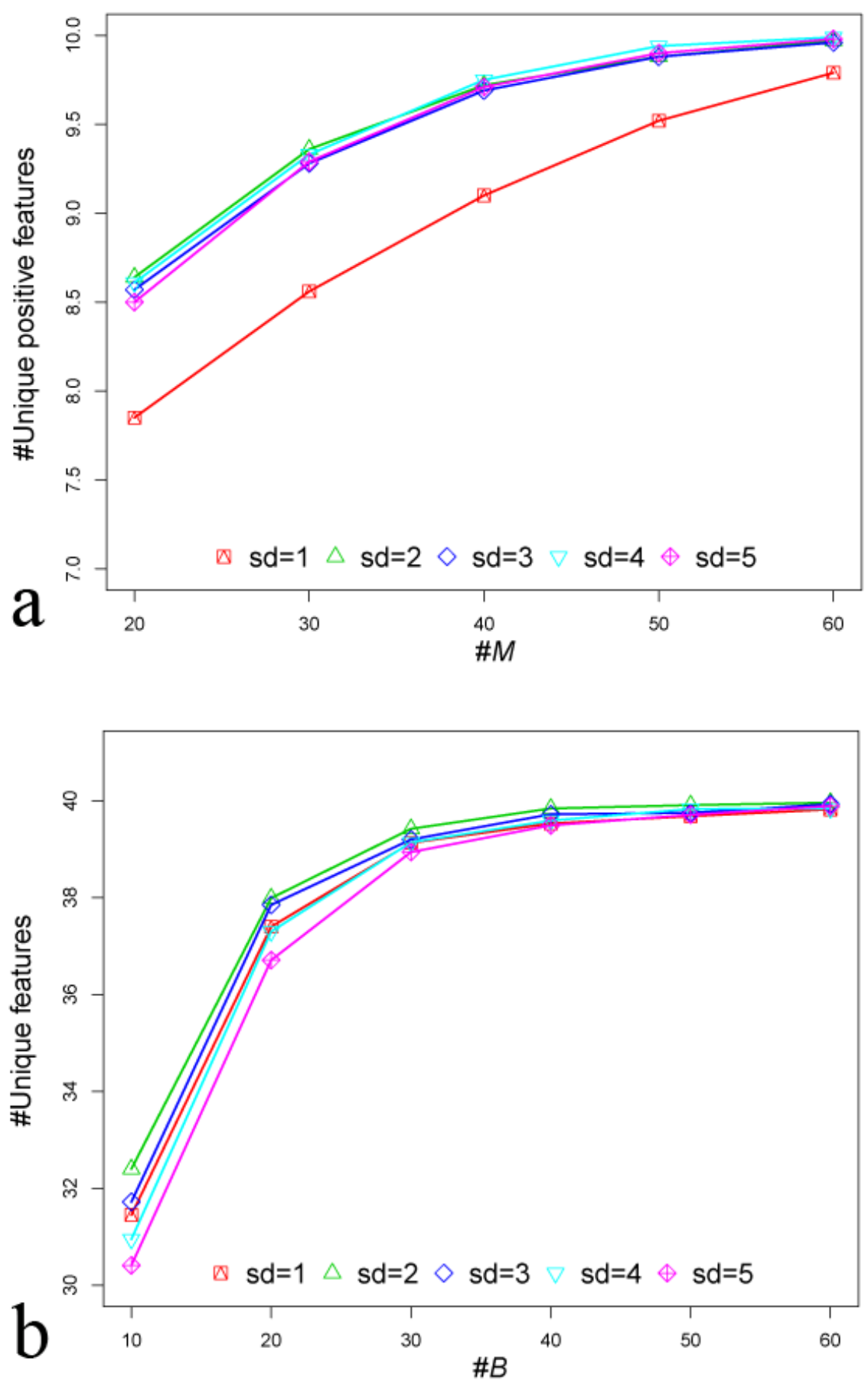


\section{5}

Comparison of results by 4 methods for synthetic dataset $S 1$ in selecting non-redundant features.

The $\mathrm{x}$-axis is the standard deviation $(s d)$ representing the parameter sds in synthetic dataset S1. The $y$-axis is the non-redundancy rate (Formula 2) in 10 selected features. The error bar represents $95 \%$ confidence interval. o 


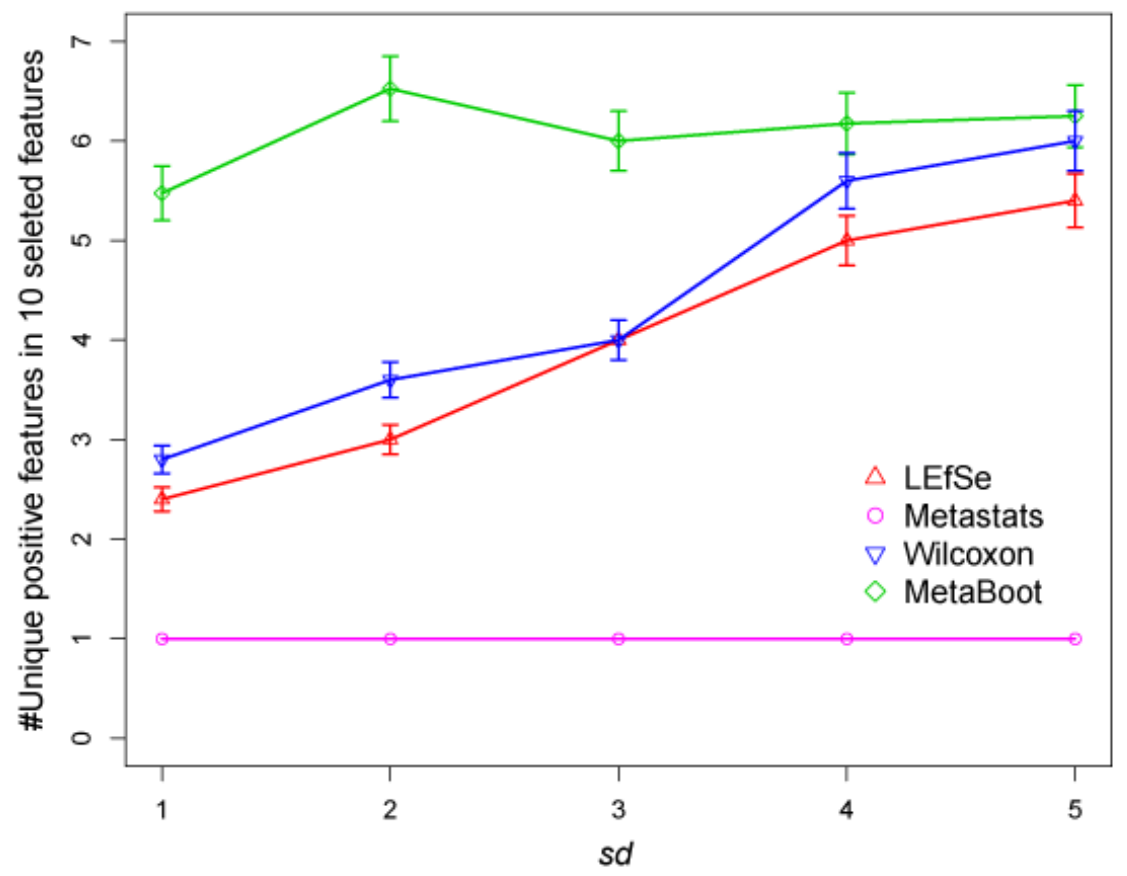


Table 3 (on next page)

Results about redundancies when applied these methods on synthetic dataset $S 2$ (Mixture dataset) and S3 (Gamma dataset) to select 10 features

Results about redundancies when applied these methods on synthetic dataset $\mathbf{S 2}$ (Mixture dataset) and S3 (Gamma dataset) to select 10 features. In columns for "LEfSe", "Metastats", "Wilcoxon" and "MetaBoot", the values were the non-redundancy rate (Formula 2) of non-redundant biomarkers with standard deviation of 1. 


\begin{tabular}{l|lllr}
\hline Dataset & LEfSe & Metastats & Wilcoxon & MetaBoot \\
\hline $\boldsymbol{S 2}$ (Mixture dataset) & $36.0 \pm 5.5$ & $26.0 \pm 5.5$ & $38.0 \pm 8.4$ & $42.0 \pm 4.5$ \\
$\boldsymbol{S 3}$ (Gamma dataset) & $46.0 \pm 11.4$ & $31.4 \pm 9.0$ & $50.0 \pm 12.2$ & $50.9 \pm 8.1$ \\
\hline
\end{tabular}

2 
Table 4(on next page)

Results about robustness when applied these methods on synthetic dataset $S 2$ (Mixture dataset) and S3 (Gamma dataset) to select 100 positive features.

Results about robustness when applied these methods on synthetic dataset 52 (Mixture dataset) and S3 (Gamma dataset) to select 100 positive features. In columns for "LEfSe", "Metastats", "Wilcoxon" and "MetaBoot", the values were "\# of positive features" with standard deviation of 1. 


\begin{tabular}{l|llll}
\hline Dataset & LEfSe & Metastats & Wilcoxon & MetaBoot \\
\hline $\boldsymbol{S 2}$ (Mixture dataset) & $67.2 \pm 2.6$ & $48.6 \pm 4.0$ & $69.0 \pm 2.5$ & $70.1 \pm 1.1$ \\
$\boldsymbol{S 3}$ (Gamma dataset) & $70.4 \pm 5.5$ & $73.3 \pm 2.9$ & $83.4 \pm 2.3$ & $81.6 \pm 2.8$ \\
\hline
\end{tabular}

2 
6

Comparison of results by 4 methods for synthetic dataset $S 1$ in selecting positive features.

The $\mathrm{x}$-axis is the standard deviation $(s d)$ representing the parameter sds in synthetic dataset $S 1$. The $y$-axis is the number of positive features in 100 selected features. The error bar represents standard deviation of $1 . R$ 


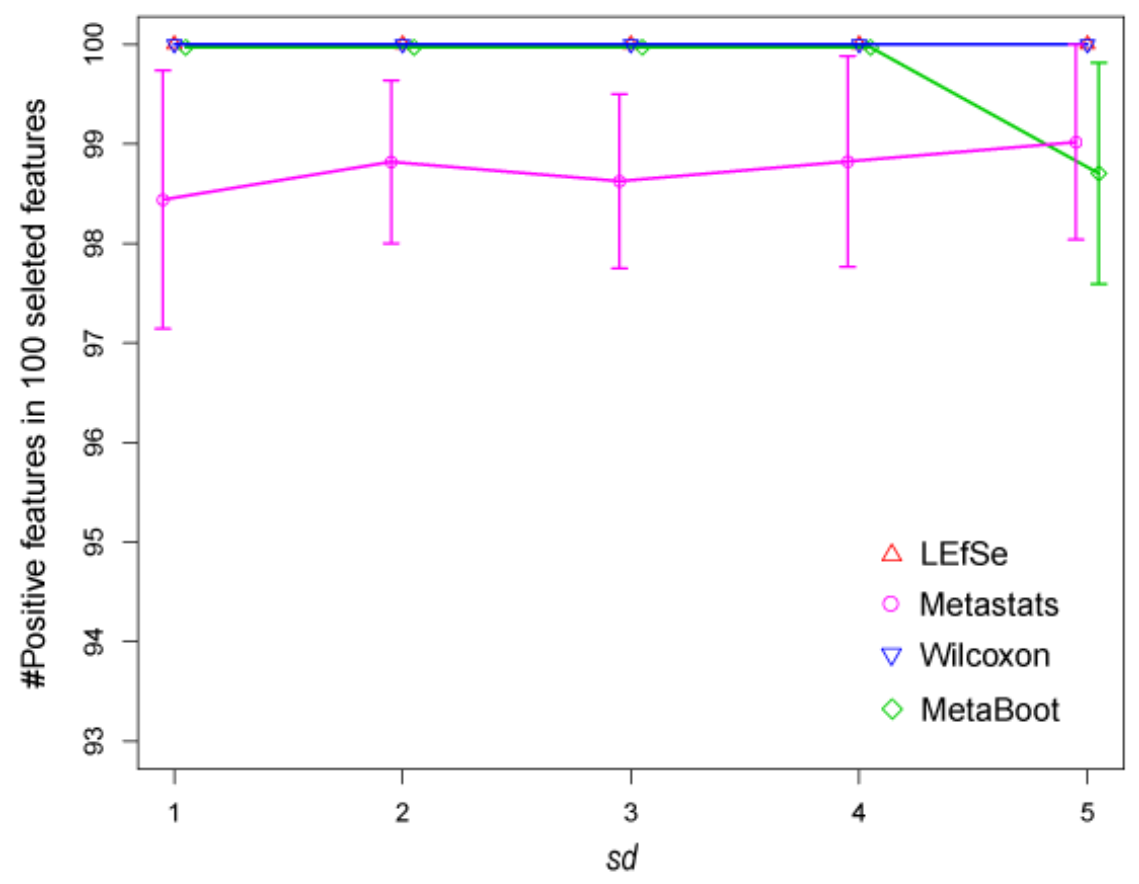


7

Comparison of accuracies when using 10 features selected by 4 methods based on synthetic dataset $S 3$.

The $x$-axis represents 4 methods and y-axis represents classification accuracy by SVM.

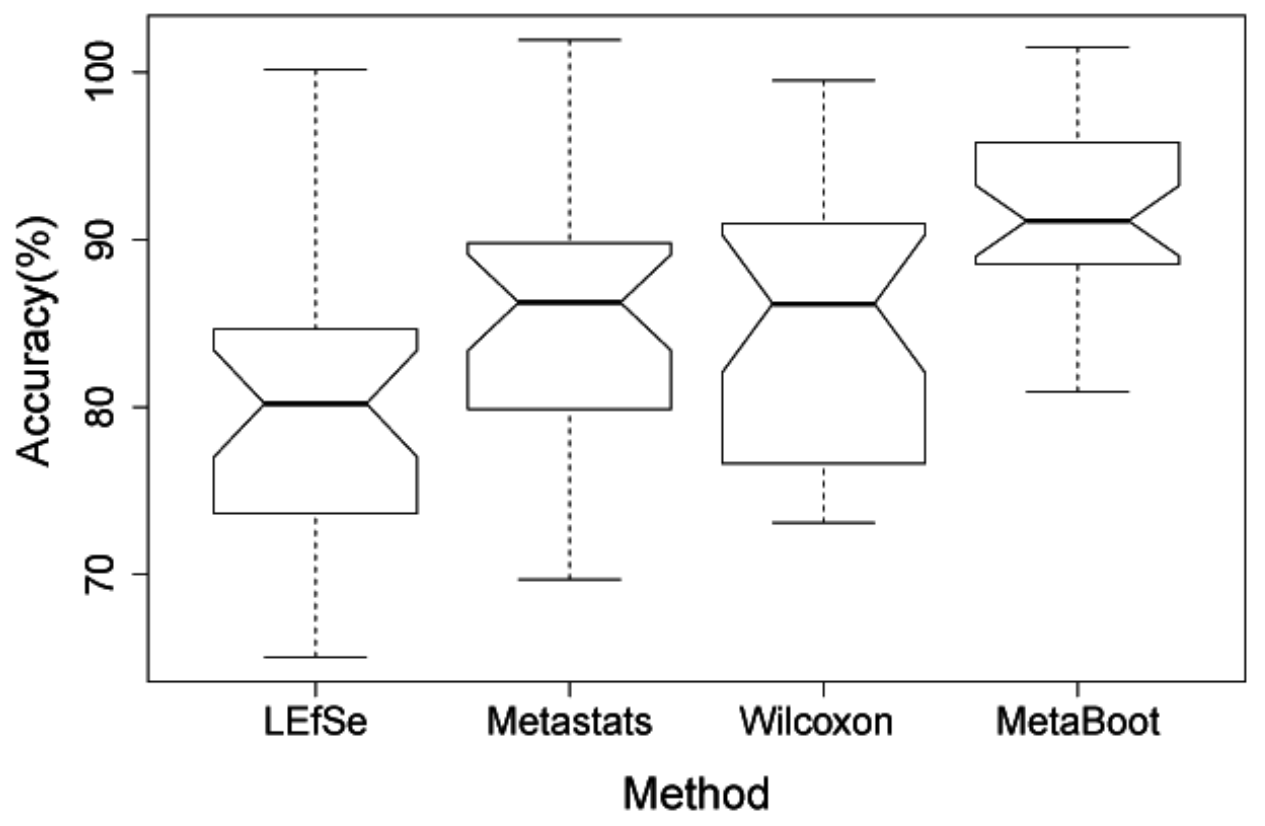


8

Biomarker identification results on oral dataset1.

(a) The Venn diagram when we selected 10 features from oral dataset1 using four methods.

(b) Circular phylogenetic tre e of oral dataset1 at genus level. The tree was generated with RAXML and viewed in ITOL (Letunic \& Bork, 2007). Genera are color-coded by phyla, except for the Firmicutes and Proteobacteria, which are shown at class level. We used the same phylogenetic tree plot from microbiome.osu.edu (Griffen et al., 2011), and we added legends onto this tree to show biomarkers selected by different methods. 

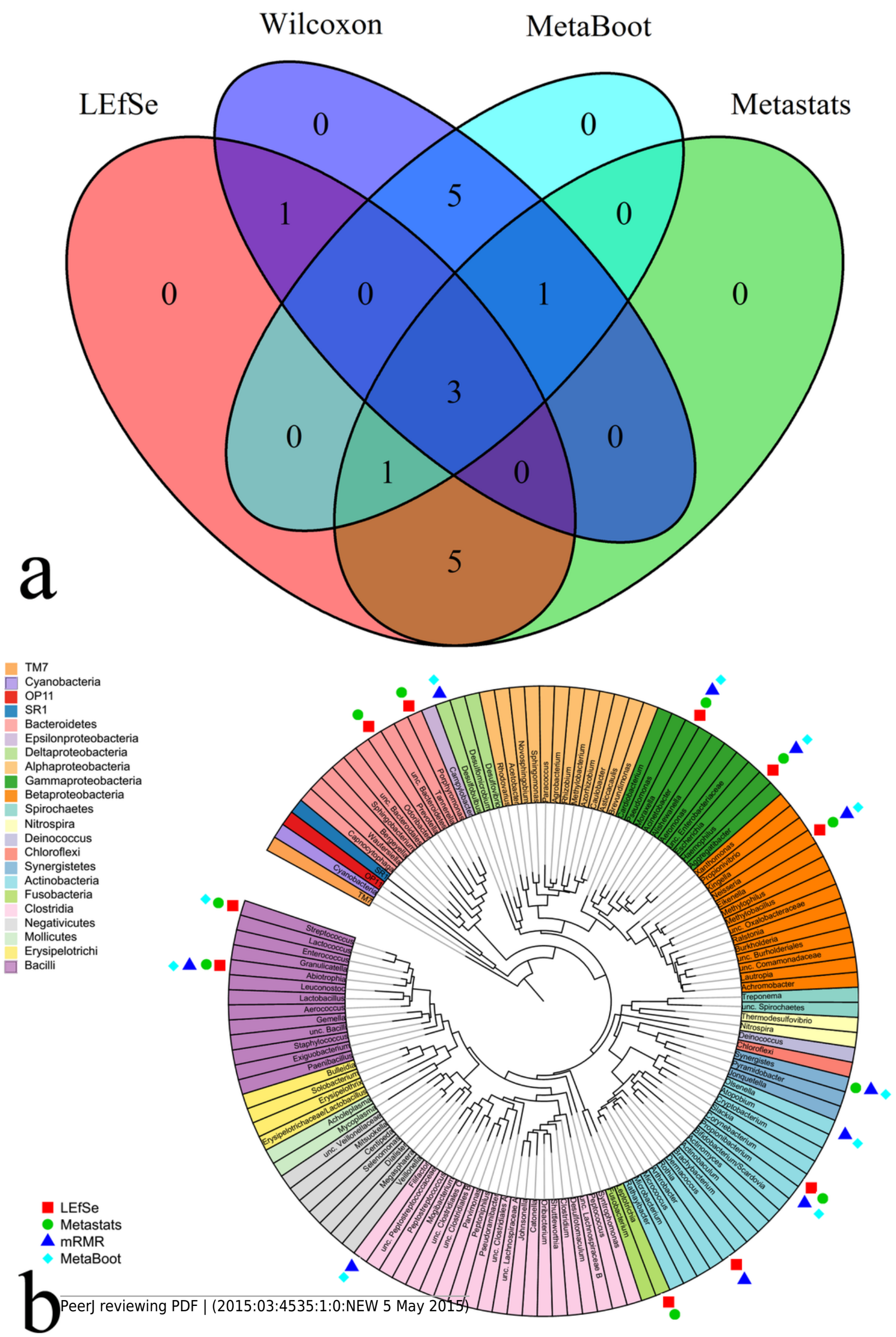
9

Comparison of accuracies when using 10 features selected by 4 methods based on oral dataset1.

The $x$-axis represents 4 methods and the $y$-axis represents the classification accuracy by SVM.

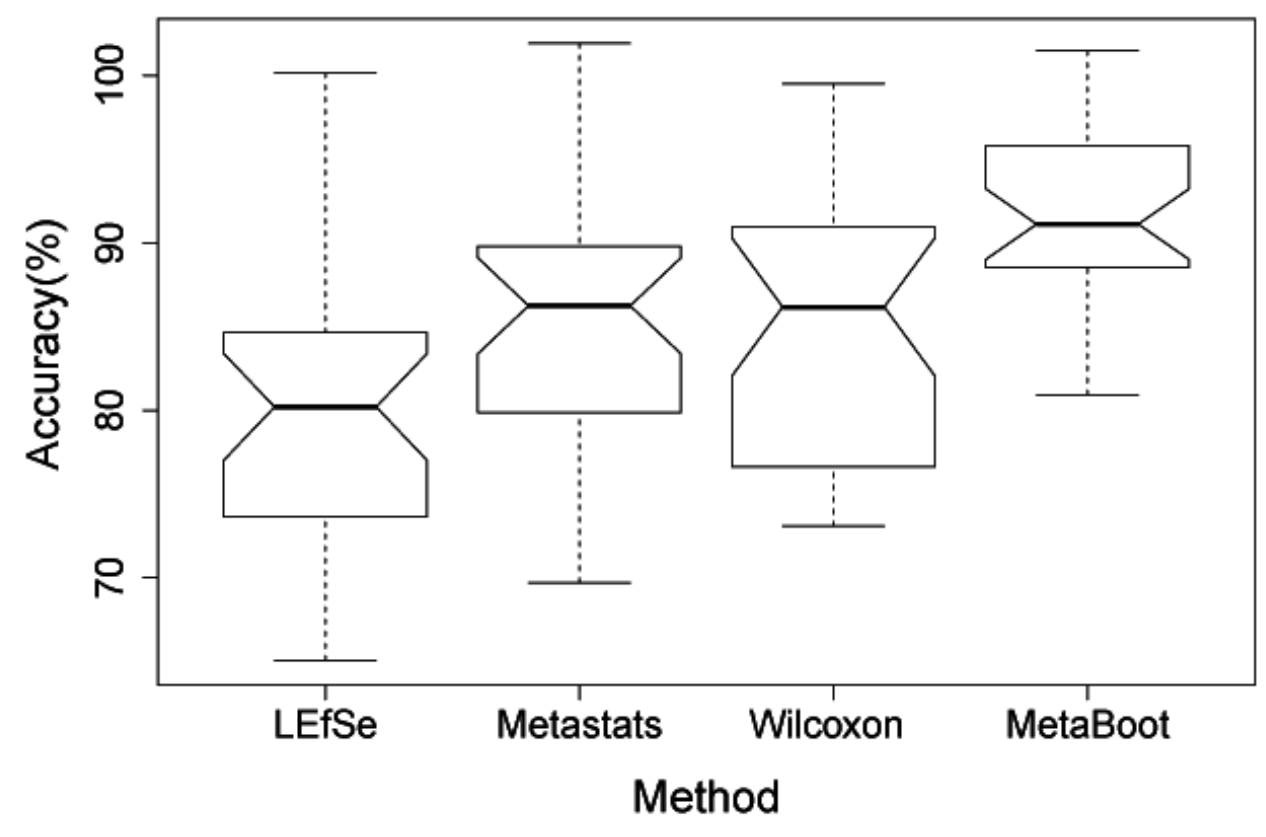




\section{0}

Biomarker identification results on oral dataset2.

(a) The Venn diagram when 10 features were selected at different level from oral dataset2

using the methods. (b) The bar-chart of average relative abundance of the features selected by MetaBoot or LEfSe and Wilcoxon. 


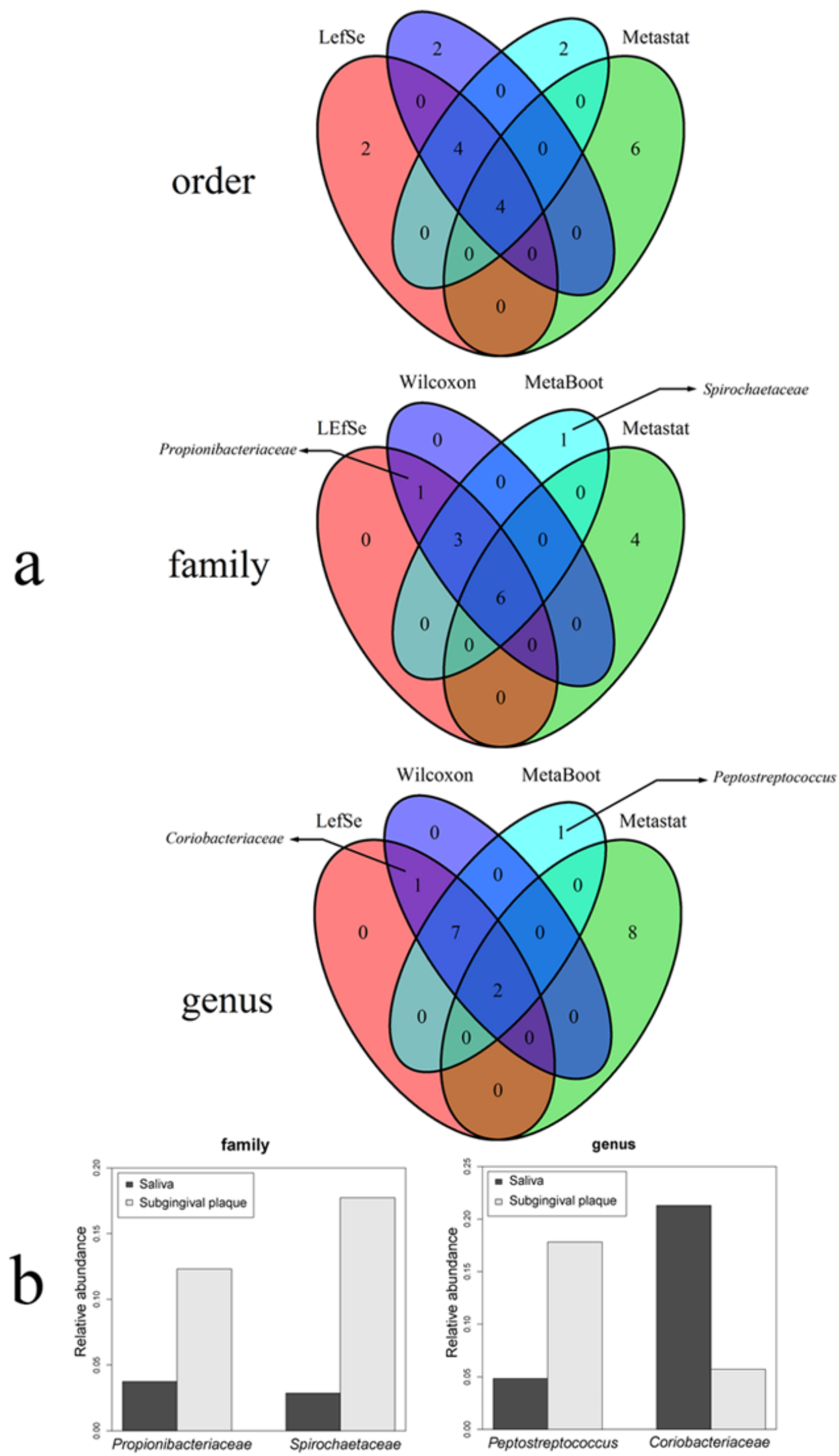


11

Biomarker identification results on soil dataset.

(a) The Venn diagram when we selected top 10 features from soil dataset using the four methods. (b) The bar-chart of average relative abundance of 5 features selected by MetaBoot under different pH values. The values for "Others" are computed as the average for other taxa. The dataset is small for standard parametric approaches. Therefore, the $p$ values $(*, 0.01 \leq p$-value $<0.05 ; * *$, p-value $<0.01)$ were calculated through permutation test s (a one-way exact test) (Kabacoff, 2011). For these five features selected, the exact test indicates a significant difference ( $p$-values are all less than 0.01 ) between two different $\mathrm{pH}$ samples. 


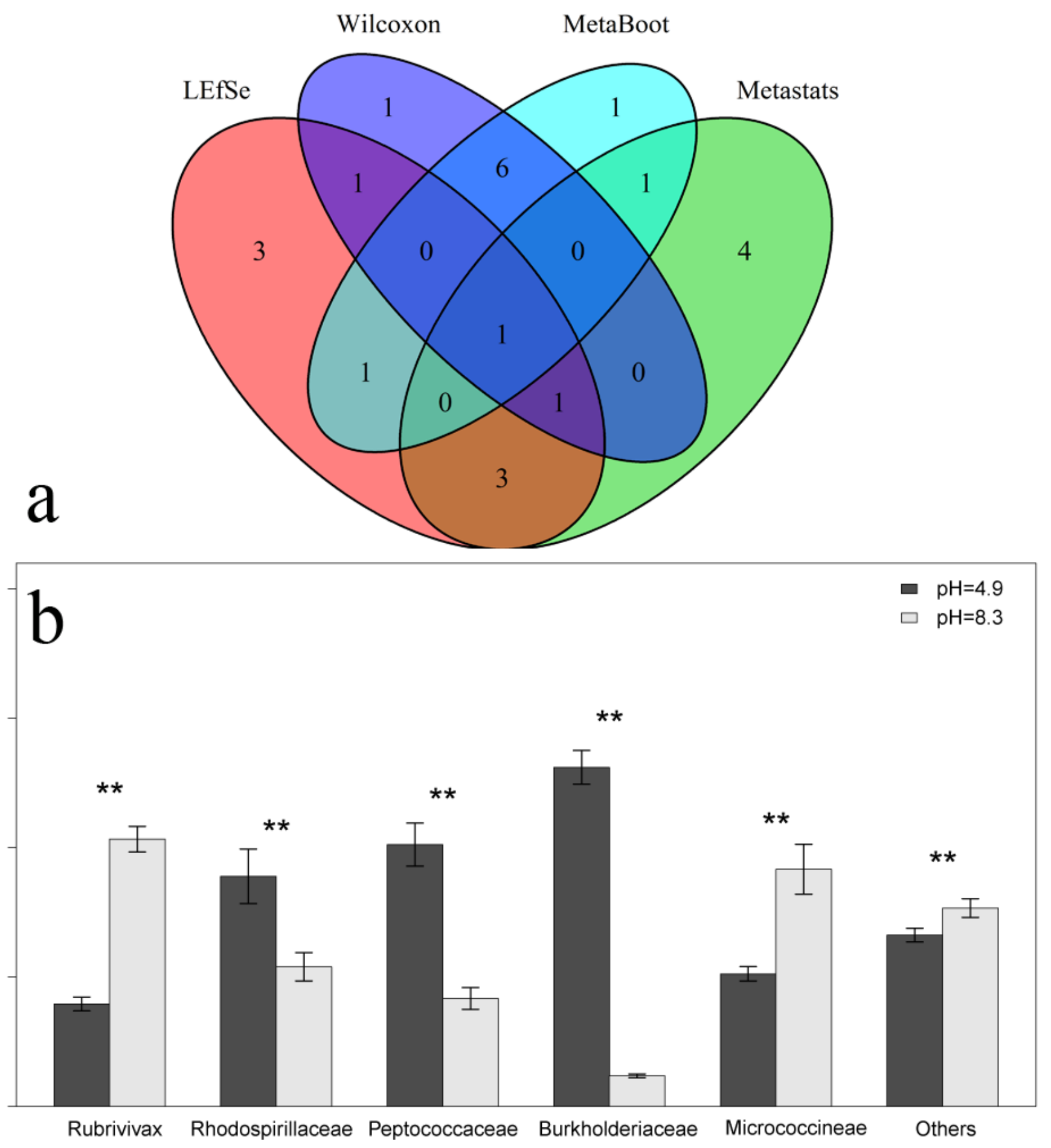

